\title{
Combustibles des ménages et modes d'utilisation à l'échelle du terroir de Vipalogo, en zone nord soudanienne du Burkina Faso
}

\author{
Georges YAMEOGO ${ }^{1}$, Barthélémy YELEMOU ${ }^{2 *}$, Lucien OUEDRAOGO ${ }^{3}$, \\ Oumar KABORE ${ }^{4}$ et Dossahoua TRAORE 5
}

\author{
${ }^{I}$ Institut de l'Environnement et de Recherches Agricoles (INERA), Cellule de Télédétection et d'Information \\ Géographique (CTIG) 01 BP 476 Ouagadougou, Burkina Faso. \\ ${ }^{2}$ Institut de l'Environnement et de Recherches Agricoles (INERA)-Saria, BP 10 Koudougou, Burkina Faso. \\ ${ }^{3}$ Institut de l'Environnement et de Recherches Agricoles (INERA) Kamboinsin, Cellule de Télédétection et \\ d'Information Géographique (CTIG) 01 BP 476 Ouagadougou, Burkina Faso. \\ ${ }^{4}$ Institut de l'Environnement et de Recherches Agricoles (INERA) Kamboinsin, Cellule de Telédetction et \\ d'Information Géographique (CTIG) 01 BP 476 Ouagadougou, Burkina Faso. \\ ${ }^{5}$ UFR Biosciences, Université de Cocody, 22 BP 582 Abidjan 22, RCI. \\ *Auteur correspondant; E- mail : yelbart@hotmail.com
}

\section{RÉSUMÉ}

Au Burkina Faso, les besoins en combustibles énergétiques sont satisfaits surtout par le bois d'énergie. En milieu rural, le bois contribue pour $95 \%$ à la satisfaction des besoins énergétiques des ménages. Une meilleure connaissance de l'importance du bois et autres combustibles dans la satisfaction des besoins et des modes de consommation du combustible permettraient une meilleure gestion des formations végétales. Pour ce faire, à l'échelle du terroir de Vipalogo, 30 exploitations ont été retenues au hasard, et enquêtées sur les combustibles énergétiques utilisés. Des mesures de consommation de combustibles ont aussi été menées régulièrement à trois périodes de l'année, période froide et sèche, saison sèche et chaude et en saison pluvieuse. A Vipalogo, les ménages utilisent $1129,8 \mathrm{~m}^{3}$ de bois par an. La consommation maximale de bois par personne et par jour atteint $1,12 \mathrm{~kg}$ en période froide contre $0,90 \mathrm{~kg}$ en période chaude et $0,67 \mathrm{~kg}$ en période pluvieuse. En outre, il ressort qu'il y a des espèces préférentielles qui répondent à un certain nombre de critères en fonction de l'usage. Si pour tous les usages, l'aspect abondance est cité, pour le bois de feu, le critère qualité de combustion est le plus signalé. Les ressources ligneuses sont prélevées à $29,6 \%$ dans les champs de cases et de brousse et le reste dans les zones non cultivées, essentiellement les savanes. Les brousses et les jachères apportent la plus grande contribution à la satisfaction des besoins des populations en ressources ligneuses. Il ressort aussi un éloignement progressif des lieux de collecte qui dénote d'une raréfaction du produit.

(C) 2014 International Formulae Group. All rights reserved.

Mots clés : combustibles énergétiques, ménage, bois, résidus de récolte, champ, jachère.

\section{INTRODUCTION}

$\mathrm{Au}$ Burkina Faso, comme dans la plupart des pays sahéliens, en milieu rural, les besoins des ménages en énergie de cuisson sont satisfaits par la biomasse, essentiellement le bois et, dans une moindre mesure, les résidus de récolte représentés par les tiges de mil ou de sorgho. La base de données énergétiques de la Direction Générale de l'Énergie (2000) montre que le bois contribue 
pour plus de $95 \%$ aux besoins énergétiques des ménages ruraux et que les résidus de récolte qui interviennent comme une énergie d'appoint, sont utilisés par plus de $75 \%$ des ménages pendant au moins trois mois dans l'année, cela juste après les récoltes. L'importance du bois de chauffe dans la satisfaction des besoins énergétiques des ménages a été signalée par plusieurs auteurs dont Gueye et Laban (1992), Bazile (1998), Loireau (1998), Gauttier et al. (2001), Edjolo et al. (2003) et Yaméogo et al. (2013). Ce qui fait que, depuis plusieurs décennies, le Sahel est confronté à de nombreuses difficultés parmi lesquelles la dégradation du couvert végétal. Cette dégradation qui a entre autres pour conséquences l'amenuisement des ressources ligneuses (Ganaba et Guinko, 1995) et la dégradation des sols, suscite, de nos jours, beaucoup d'inquiétudes quant aux possibilités de promouvoir un développement durable au bénéfice des populations. Cela est d'autant plus réel que, le domaine non cultivé, à savoir, les savanes et les jachères qui constituent les réserves de terres cultivables, sont également les lieux par excellence de la collecte de bois pour la satisfaction des besoins énergétiques ce qui leur confère le statut d'entités utiles comme le soulignent Le Roy et al. (1996).

$\mathrm{Au}$ regard des conséquences engendrées par la forte consommation de bois sur les potentialités des ressources forestières, plusieurs institutions sous régionales et internationales fournissent aux États sahéliens, des appuis financiers pour la mise en œuvre de programmes de réduction de la consommation de bois et de reconstitution du couvert végétal.

Malgré ces efforts, les ressources ligneuses ne font que s'amenuiser et il n'y a pas pour le moment, surtout en milieu rural, une alternative à l'utilisation du bois pour la satisfaction des différents besoins en matière de bois de chauffe.
Ainsi, les objectifs fondamentaux auxquels répond la présente étude, menée dans le terroir de Vipalogo au Burkina Faso, sont de connaître la consommation de bois de feu et de résidus de récolte en site réel d'utilisation. L'ensemble de ces données devra permettre d'estimer les besoins en bois de feu, estimation intéressante pour l'appréciation des impacts de leurs prélèvements sur les ressources ligneuses pour une prise de conscience des populations.

\section{MATERIEL ET METHODES}

\section{Localisation et caractérisation du site}

Le site de l'étude est Vipalogo, un village situé à $40 \mathrm{~km}$ au Sud-Ouest de Ouagadougou (capitale du Burkina Faso et chef-lieu de la province du Kadiogo). Il est compris entre la latitude $12^{\circ} 04^{\prime} 20^{\prime}$ ' et $12^{\circ} 09^{\prime} 30^{\prime \prime}$ nord et $1^{\circ} 42^{\prime} 50^{\prime}$, et $1^{\circ} 49^{\prime} 00^{\prime \prime}$ ouest (Figure 1).

Le terroir de Vipalogo fait partie du grand ensemble généralement appelé «Plateau Central» qui se caractérise par une forte concentration humaine de 76 habitants au $\mathrm{km}^{2}$, contre une moyenne nationale de 38 habitants au $\mathrm{km}^{2}$ (INSD, 1998). On note aussi une intense occupation qui entraîne la dégradation des terres et des conflits assez fréquents entre agriculteurs et entre agriculteurs et éleveurs. Vipalogo appartient au domaine phytogéographique NordSoudanien (Fontes \& Guinko, 1995) caractérisé par des savanes arborées et arbustives à Vitellaria paradoxa Gaertn.f.. Lannea microcarpa Engl. \& K. Krause, Parkia biglobosa (Jacq.) R.Br.exG.Don., Faidherbia albida (Del.) Chev., Tamarindus indica L., Combretum micranthum G. Don, Combretum glutinosum Perr. ex DC. , Guiera senegalensis J.F.Gmel., Acacia seyal Del. Le paysage est fortement marqué par l'emprise humaine, illustrée par la présence de savanes parcs et de jachères à karité (Vitellaria paradoxa) et à néré (Parkia biglobosa). 
Le climat est caractérisé par une saison sèche de sept mois (de novembre à mai) et une saison pluvieuse de cinq mois (de juin à octobre). La pluviométrie annuelle est très variable et oscille entre $600 \mathrm{~mm}$ et $1000 \mathrm{~mm}$, avec une moyenne de $761 \mathrm{~mm}$ sur les trente dernières années. Les températures moyennes mensuelles connaissent également une variation allant de $24^{\circ} \mathrm{C}$ à $33^{\circ} \mathrm{C}$.

\section{Méthodologie}

A la suite du recensement de la population (Yaméogo, 2009), 409 ménages répartis dans cinq quartiers ont été identifiés dans le terroir de Vipalogo. Sur la base de la typologie des exploitations agricoles du terroir, il a été mis en évidence trois grands groupes caractérisés comme suit :

(i) le groupe exploitant une superficie agricole comprise entre 1 ha et 3 ha, avec un faible niveau d'équipement agricole ( 1 houe manga), une faible pratique de l'élevage (moins de 10 têtes de bovins et caprins). Ce groupe constitue $93 \%$ des exploitations du terroir;

(ii) le groupe exploitant une superficie agricole comprise entre 3 ha et 6 ha, avec un niveau moyen d'équipement agricole ( 1 à 2 houes manga et 1 charrette) et une pratique moyenne de l'élevage (10 à 20 têtes de bovins et des caprins). Il représente $4,41 \%$ des exploitations et;

(iii) le groupe exploitant plus de 6 ha de superficie agricole avec un bon niveau d'équipement agricole ( 2 houes manga, 1 charrette et 1 charrue) et une grande pratique de l'élevage (plus de 20 têtes de bovins, des ovins et des caprins) ce groupe constitue 2,59\% des exploitations.

$\mathrm{Au}$ sein de chaque quartier, 6 exploitations agricoles ont été retenues de manière aléatoire et simple (en utilisant le logiciel SPSS) dans le groupe représentant
93\% des ménages soit un taux d'échantillonnage de 7,4\%.

Afin de cerner les effets des variations saisonnières sur la consommation de combustible, les mesures de consommation ont été effectuées à trois périodes de l'année: en période sèche et froide (janvier), en saison sèche et chaude (mai) et en saison pluvieuse (août). La prise en compte de ces périodes a permis, entre autres, de tenir compte de l'utilisation des résidus de récolte pour la satisfaction des besoins énergétiques à certaines périodes de l'année.

Pour tenir compte de la méthodologie utilisée, les résultats ont été exprimés en consommation par personne et par jour. La consommation (Conso) de bois par personne (Pers) et par jour en kilogramme pour une période donnée est exprimée par la formule suivante:

$$
\text { ConsoBoisPersJour } \mathrm{j}=\frac{\sum_{1}^{30} \text { ConsoBoisPersJour } \mathrm{i}}{30}
$$

La moyenne annuelle de consommation a été calculée en faisant la moyenne des consommations des périodes considérées, suivant la formule ci-dessous:

ConsoBoisPersJour $=\frac{\sum_{1}^{3} \text { ConsoBoisPersJour } \mathrm{J}}{3}$

Les mêmes formules ont été utilisées pour les résidus de récolte en remplaçant le bois par les résidus de récolte.

Une analyse de variance a été faite afin de comparer l'effet de la période de l'année (période sèche et froide, période sèche et chaude, période pluvieuse) sur la consommation de bois ainsi que sur la consommation des résidus de récolte (tiges de mil) des ménages du terroir de Vipalogo. 


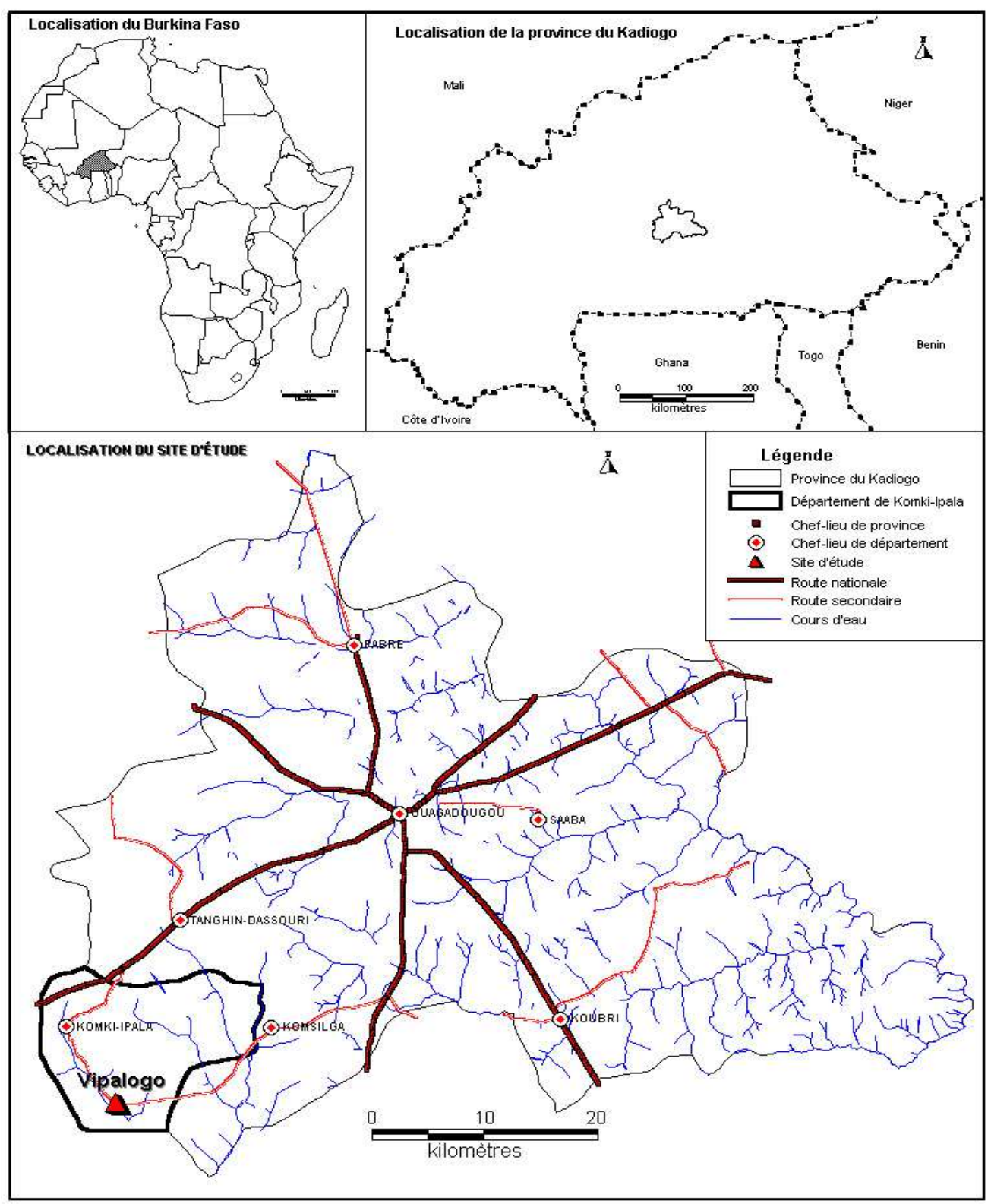

Figure 1 : Localisation du site de Vipalogo.

\section{RÉSULTATS}

Les combustibles, d'approvisionnement, périodicité et espèces préférées

Les résultats de nos enquêtes montrent que $93,33 \%$ des ménages utilisent le bois comme source d'énergie principale (Tableau
1) et $6,67 \%$, utilisent la bouse de vache pour la cuisson des repas. Ces ménages utilisent donc soit le bois comme source d'énergie principale soit les résidus de récolte en source d'énergie secondaire.

A Vipalogo, le ramassage est le mode d'approvisionnement en bois le plus utilisé 
par tous les ménages. Toutefois, environ $10 \%$ complètent souvent leurs besoins par l'achat. Le ramassage du bois pour la préparation des repas incombe aux femmes et aux enfants et le mode de transport est le port sur la tête. 3,34\% utilise la charrette à traction animale.

Le lieu d'approvisionnement en bois de chauffe est très variable avec une forte représentativité des brousses et des jachères (Figure 2). La notion de brousse recouvre les vieilles jachères présentes aujourd'hui sous forme de savanes arbustives avec une prédominance des Combretaceae telles que Guiera senegalensis, Terminalia avicennioides, Terminalia macroptera. Le bois de feu est surtout prélevé dans les zones non cultivées (brousses et jachères).

La périodicité d'approvisionnement est variable et nos enquêtes révèlent que la grande majorité des ménages (36,66\%), s'approvisionne une fois tous les deux jours ou une fois par semaine. Ils sont seulement $4 \%$ des ménages à s'approvisionner tous les quatre jours et $10 \%$ tous les quinze jours. Cependant, $86,66 \%$ de la population estiment qu'il y a des difficultés actuellement pour s'approvisionner en bois de chauffe. L'éloignement des zones d'approvisionnement est la principale difficulté citée par les populations. Ensuite vient la recherche d'espèces particulières donnant du bon bois de feu et qui deviennent de plus en plus rares. En effet, l'enquête montre que les populations ont des préférences par rapport aux espèces à utiliser comme bois de feu. Les espèces recherchées pour être utilisées comme bois de feu sont consignées dans le Tableau 2.

Dans le terroir, certaines espèces ligneuses ne sont pas utilisées comme bois de feu. C'est le cas de Calotropis procera (Asclepiadaceae), Tamarindus indica (Bombacaceae), Bombax costatum (Bombacaceae), Annona senegalensis (Annonaceae), Afzelia africana (Caesalpiniaceae), Entada africana (Mimosaceae), Sclerocarya birrea (Anacardiaceae), Daniellia oliveri
(Caesalpiniaceae) et Stereospermum kunthianum (Bignoniaceae). Les raisons évoquées sont liées soient aux croyances (arbres habités par de génies) ou au fait que leur utilisation comme bois de feu engendre des maladies (maux d'yeux, maladies de la peau, vertiges).

De l'identification des types d'équipement utilisés pour la cuisson, à Vipalogo, il ressort que tous les ménages enquêtés utilisent le système traditionnel représenté par le foyer traditionnel à trois pierres (Photo 1). Toutefois, 6,67\% des ménages possèdent des foyers améliorés (Photo 2) et 13,33\% utilisent des foyers traditionnels de type métallique en deuxième choix.

\section{Evaluation de la consommation de bois}

Les valeurs moyennes de la consommation de bois sont variables suivant les périodes de l'année (Tableau 3).

Les plus fortes variations de la consommation de bois sont observées pendant la période froide, comme le montre la valeur de l'écart type. En outre, l'analyse statistique révèle des différences statiques dans la consommation des ménages selon les saisons (Tableau 4).

L'analyse de variance indique que le $\mathrm{Pr}$ $>\mathrm{F}$ est inférieur à $5 \%$. Avec $5 \%$ de marge d'erreur, au moins deux des trois moyennes sont significativement différentes entre elles. Cette situation a amené à procéder à une comparaison des moyennes de consommation suivant les périodes par le test $\mathrm{t}$ de Student au seuil de 95\% (Tableau 5). Ce tableau montre que les moyennes de la période froide et celle de la période chaude ne sont pas significativement différentes. Par contre, les moyennes du mois froid et du mois chaud sont significativement différentes de celle du mois représentant la période pluvieuse.

Il existe en outre une variabilité de la consommation de bois par personne et par jour en fonction des saisons (Figure 3). Il ressort de la Figure 3 que la consommation maximale peut atteindre $1,12 \mathrm{~kg}$ en période 
froide, $0,90 \mathrm{~kg}$ en période chaude et $0,67 \mathrm{~kg}$ en période pluvieuse. Quant aux valeurs minimales, elles sont respectivement de 0,36 $\mathrm{kg} ; 0,42 \mathrm{~kg}$ et $0,37 \mathrm{~kg}$ pour les mêmes périodes. Le calcul du coefficient de variation donne une valeur de $42,1 \%$.

Sur la base de ces trois valeurs moyennes saisonnières, il résulte que la consommation annuelle moyenne par personne et par jour à Vipalogo est de $0,64 \mathrm{~kg}$ avec un écart type de $0,27 \mathrm{~kg}$, soit un besoin annuel par personne de 233,6 kg. En extrapolant à la population totale du terroir qui est de 3869 personnes, les besoins annuels totaux sont estimés à 903,80 tonnes soit 1130 $\mathrm{m}^{3}$. L'ajustement de ces valeurs par une courbe de régression (Figure 4) donne l'équation (y) suivante : $y=0,0184 x^{2}-0,2962 x$ $+1,7054$ pour la consommation de bois en fonction de la taille du ménage avec un coefficient de corrélation $\mathrm{R}^{2}=47,69 \%$. La valeur du coefficient de corrélation montre que la taille du ménage n'a pas beaucoup d'influence sur la consommation de bois à Vipalogo. Elle explique seulement pour $48 \%$, la consommation moyenne de bois par personne et par jour. L'évolution de la consommation moyenne par personne et par jour selon la taille du ménage, présente une grande variabilité.

\section{Evaluation de la consommation de résidus de récolte}

Les résidus de récoltes sont surtout utilisés au cours de la période froide. Leur utilisation par les ménages chute de la période froide à la période pluvieuse ou elle est nulle. L'analyse de la variance montre que le $\mathrm{Pr}>\mathrm{F}$ est inférieur à $1 \%$, donc au moins deux des trois moyennes sont très significativement différentes entre elles au seuil de 95\%. Le test t de Student montre que les moyennes de la période chaude et celle de la période pluvieuse ne sont pas significativement différentes (Tableau 5). Par contre, la moyenne de la période froide est significativement supérieure à celle de la période chaude.

En outre, il apparaît une grande variabilité de la consommation de résidus de récolte par personne et par jour en fonction des saisons (Figure 5). La consommation maximale peut atteindre $0,52 \mathrm{~kg}$ en période froide, 0,04 $\mathrm{kg}$ en période chaude. Quant aux valeurs minimales, elles sont respectivement de $0,14 \mathrm{~kg}$ pour la période froide et nulle pour les autres périodes. Sur la base de ces trois valeurs moyennes par période, il ressort que la consommation de résidus de récolte par personne et par jour à Vipalogo est de $0,1 \mathrm{~kg}$. Le calcul du coefficient de variation donne une valeur de 98,38\%. L'évolution de la consommation des résidus de récolte selon la taille du ménage (Figure 6) est de la forme :

$$
\mathrm{y}=0,0025 \mathrm{x}^{2}-0,0423 \mathrm{x}+0,2776 \text { avec }
$$

un coefficient de corrélation $\mathrm{R}^{2}=16,8 \%$.

Les besoins moyens en bois des ménages de Vipalogo par personne et par jour sont de 0,64 kg avec un écart type de 0,27 kg tandis que pour les résidus de récolte, ces besoins sont de $0,10 \mathrm{~kg}$ avec un écart type de $0,11 \mathrm{~kg}$. On note pour ces deux combustibles de fortes variations autour de la moyenne.

Tableau 1: Sources d'énergie utilisées pour la cuisson.

\begin{tabular}{lccc}
\hline & Source principale $\mathbf{( \% )}$ & Deuxième source $\mathbf{( \% )}$ & Troisième source $\mathbf{( \% )}$ \\
\hline Bois de feu & 93,33 & 6,67 & 3,33 \\
Résidus de cultures & 0 & 93,33 & 0 \\
Bouses de vaches & 6,67 & 0 & 0 \\
Autre & 0 & 0 & 6,67 \\
Sans réponse & 0 & 0 & 3,33 \\
\hline
\end{tabular}


Tableau 2: Principales espèces utilisées comme bois de feu.

\begin{tabular}{lccc}
\hline Espèces & $\begin{array}{c}\text { Premier } \\
\text { choix } \mathbf{( \% )}\end{array}$ & Deuxième choix (\%) & $\begin{array}{c}\text { Troisième choix } \\
(\%)\end{array}$ \\
\hline Erythrina senegalensis DC. & 33,33 & 26,67 & 20 \\
Detarium microcarpum Guill. \& Perr. & 16,67 & 26,67 & 0,00 \\
Vitellaria paradoxa Gaertn. f. & 13,33 & 6,67 & 3,33 \\
Acacia seyal Del. & 13,33 & 0,00 & 0,00 \\
Parkia biglobosa (Jacq.) Benth. & 6,67 & 13,33 & 6,67 \\
Mangifera indica L. & 6,67 & 0,00 & 0,00 \\
Guiera senegalensis J. F. Gmel. & 3,33 & 10 & 36,67 \\
Piliostigma reticulatum (DC.) Hochst. & 3,33 & 3,33 & 6,67 \\
Burkea africana Hook. & 3,33 & 3,33 & 0,00 \\
Cassia sieberiana DC. & 0,00 & 3,33 & 3,33 \\
Terminalia avicennioides Guill. \& Perr. & 0,00 & 3,33 & 3,33 \\
Azadirachta indica A. Juss. & 0,00 & 3,33 & 0,00 \\
Acacia senegal (L.) Willd. & 0,00 & 0,00 & 6,67 \\
Lannea microcarpa Engl. \& K. Krause & 0,00 & 0,00 & 3,33 \\
Faidherbia albida Del. & 0,00 & 0,00 & 3,33 \\
\hline
\end{tabular}

Tableau 3: Consommation moyenne de bois et de résidus de récolte par personne et par jour en fonction des périodes de l'année.

\begin{tabular}{lcccc}
\hline & $\begin{array}{c}\text { Consommation moyenne de } \\
\text { résidus de récolte }\end{array}$ & \multicolumn{2}{c}{$\begin{array}{c}\text { Consommation moyenne de } \\
\text { résidus de récolte }\end{array}$} \\
\hline Période de l'année & $\begin{array}{c}\text { Cons/Pers./jour } \\
\text { en kg }\end{array}$ & Ecart type & $\begin{array}{c}\text { Cons/Pers./jour } \\
\text { en kg }\end{array}$ & Ecart type \\
\hline Période froide & 0,73 & 0,37 & 0,33 & 0,19 \\
Période chaude & 0,66 & 0,24 & 0,01 & 0,04 \\
Période pluvieuse & 0,52 & 0,15 & 0,00 & 0,00 \\
Moyenne annuelle & 0,64 & 0,27 & 0,1 & 0,11 \\
\hline
\end{tabular}

Tableau 4: Analyse de variance de la consommation selon les types de combustibles.

\begin{tabular}{lcccccc}
\hline $\begin{array}{l}\text { Types } \\
\text { combustibles }\end{array}$ & $\begin{array}{c}\text { Source de } \\
\text { variation }\end{array}$ & $\begin{array}{c}\text { Degrés } \\
\text { de } \\
\text { liberté. }\end{array}$ & $\begin{array}{c}\text { Somme } \\
\text { des carrés } \\
\text { des écarts }\end{array}$ & $\begin{array}{c}\text { Carrés } \\
\text { moyens }\end{array}$ & F observé & Pr $>$ F \\
\hline Bois & Variance factorielle & 2 & 0,66 & 0,33 & 4,62 & 0,0124 \\
& Variance résiduelle & 87 & 6,25 & 0,07 & & \\
& Total & 89 & 6,92 & & & \\
\hline Résidus de & Variance factorielle & 2 & 2,10 & 1,05 & 87,35 & $<0,0001$ \\
récolte & Variance résiduelle & 87 & 1,04 & 0,01 & & \\
& Total & 89 & 3,13 & & & \\
\hline
\end{tabular}


Tableau 5 : Comparaison des moyennes de consommation de bois en fonction des périodes de l'année.

\begin{tabular}{lcccc}
\hline Type combustible & t Groupe & $\begin{array}{c}\text { Moyenne } \\
\text { en kg }\end{array}$ & N & CBMJ \\
\hline Bois & A & 0,73 & 30 & CBMJ1 \\
& A & 0,66 & 30 & CBMJ5 \\
& B & 0,52 & 30 & CBMJ8 \\
\hline Résidus de récolte & A & 0,33 & 30 & CTMMJ1 \\
& A & 0,01 & 30 & CTMMJ5 \\
& B & 0,00 & 30 & CTMMJ8 \\
\hline CBMJ1 - Consommation de bois moyen par jour pendant la période froide; & \\
CBMJ5 - Consommation de bois moyen par jour pendant la période chaude; & \\
CBMJ8 - Consommation de bois moyen par jour pendant la période pluvieuse; \\
CTMMJ1 - Consommation moyenne de tige de mil par jour et par personne pendant la période froide; \\
CTMMJ - Consommation moyenne de tige de mil par jour et par personne pendant la période chaude; \\
CTMMJ8 - Consommation moyenne de tige de mil par jour et par personne pendant la période pluvieuse; \\
CTMMJ - Consommation moyenne de tige de mil par jour et par personne pendant la période chaude; \\
CTMMJ8 - Consommation moyenne de tige de mil par jour et par personne pendant la période pluvieuse.
\end{tabular}

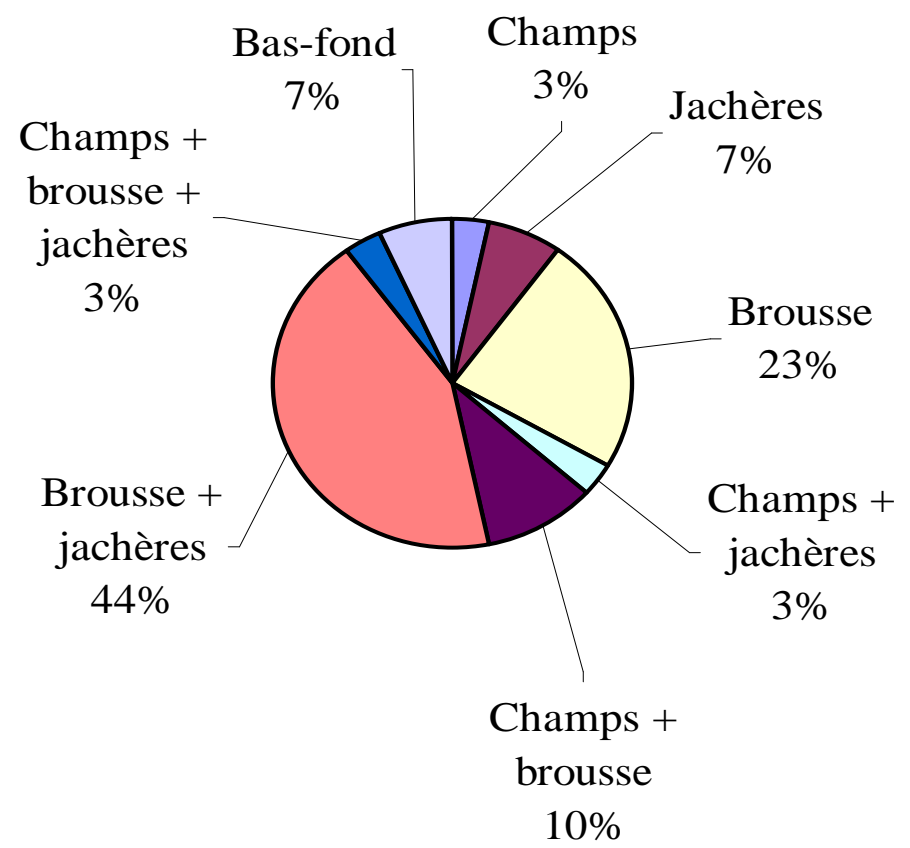

Figure 2 : Lieu d'approvisionnement en bois de chauffe. 


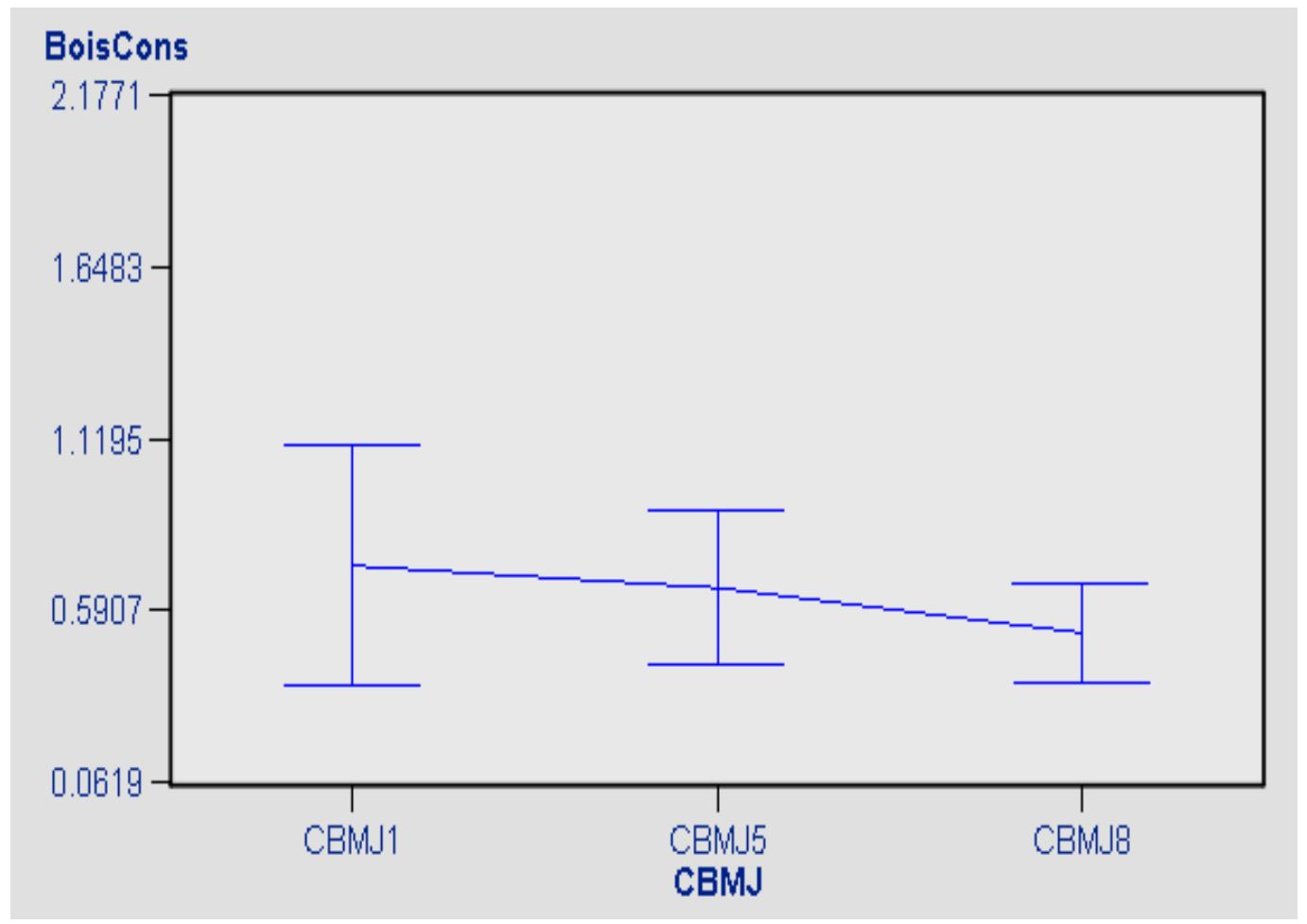

Figure 3 : Variation de la consommation de bois en fonction des périodes de l'année.

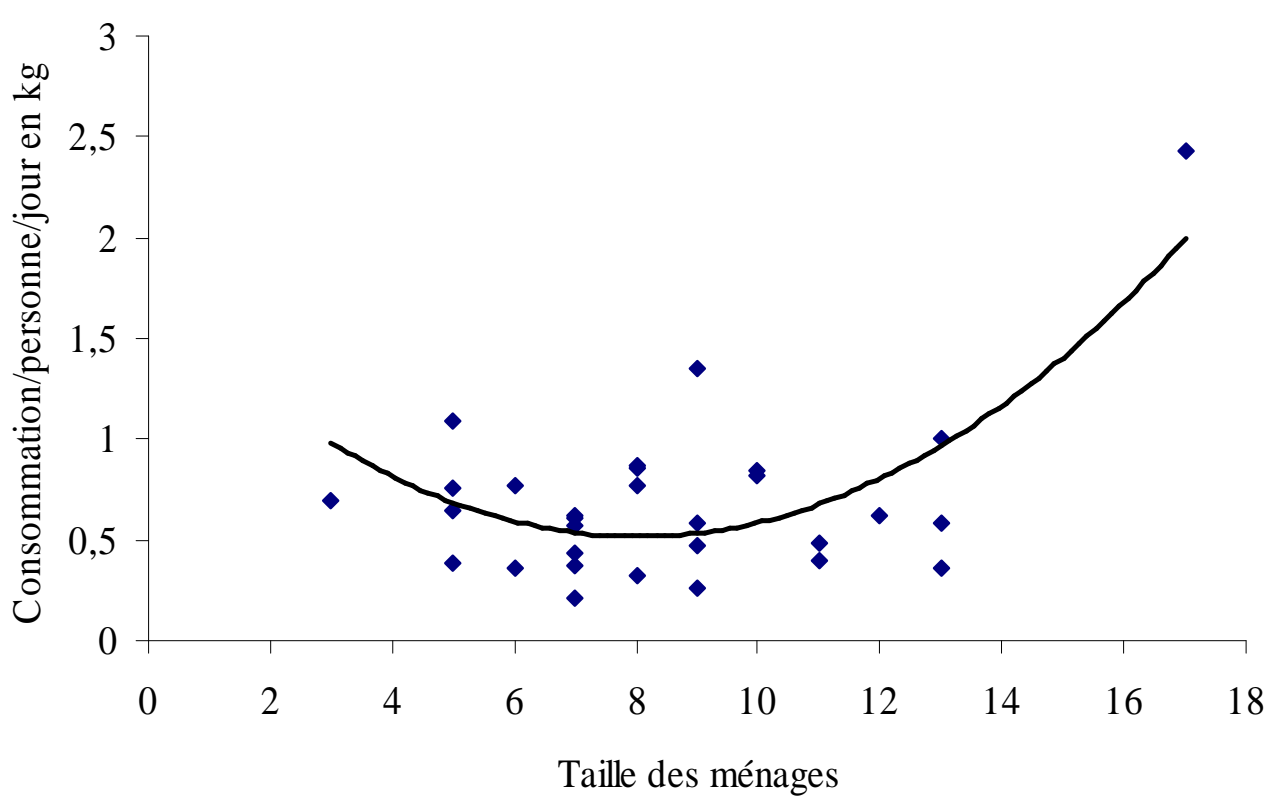

Figure 4 : Consommation de bois en fonction de la taille du ménage. 


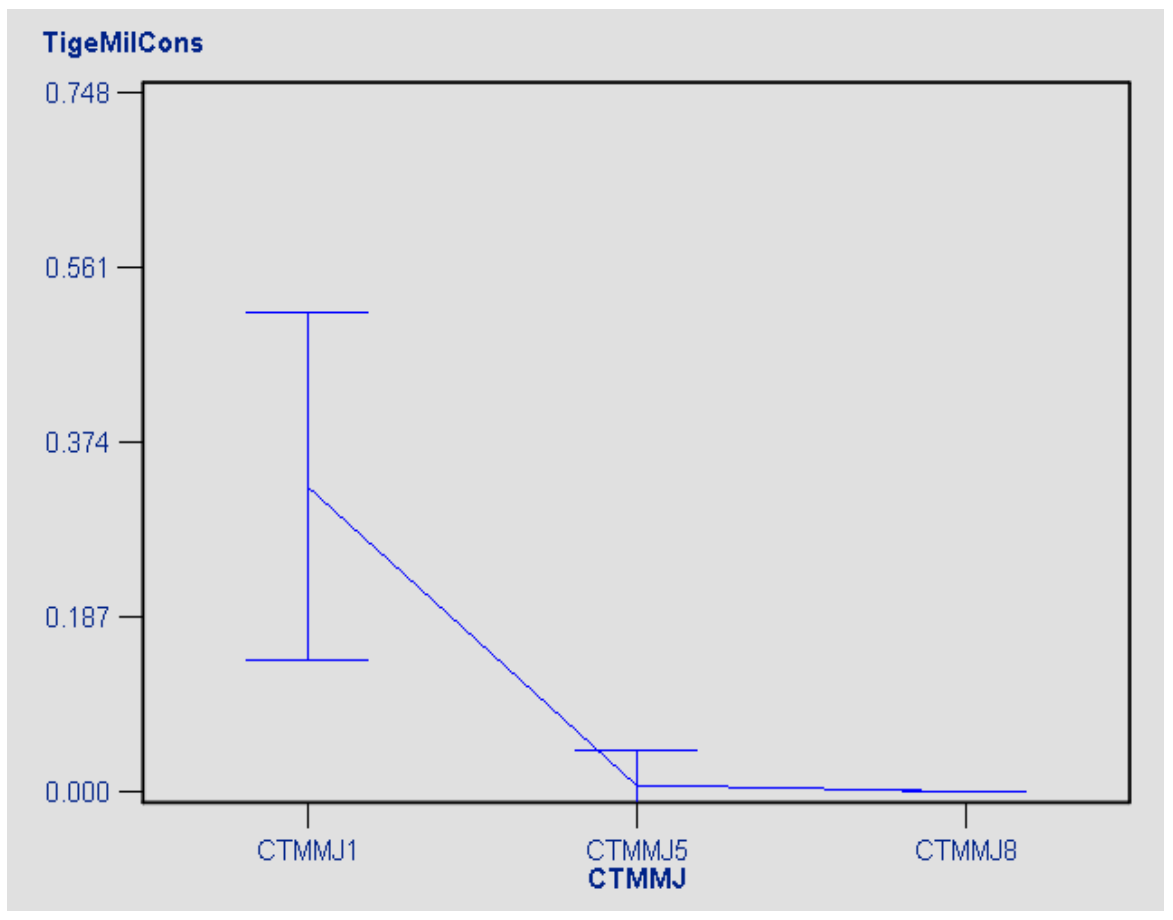

Figure 5 : Variation de la consommation de résidus de récolte en fonction des périodes de l'année.

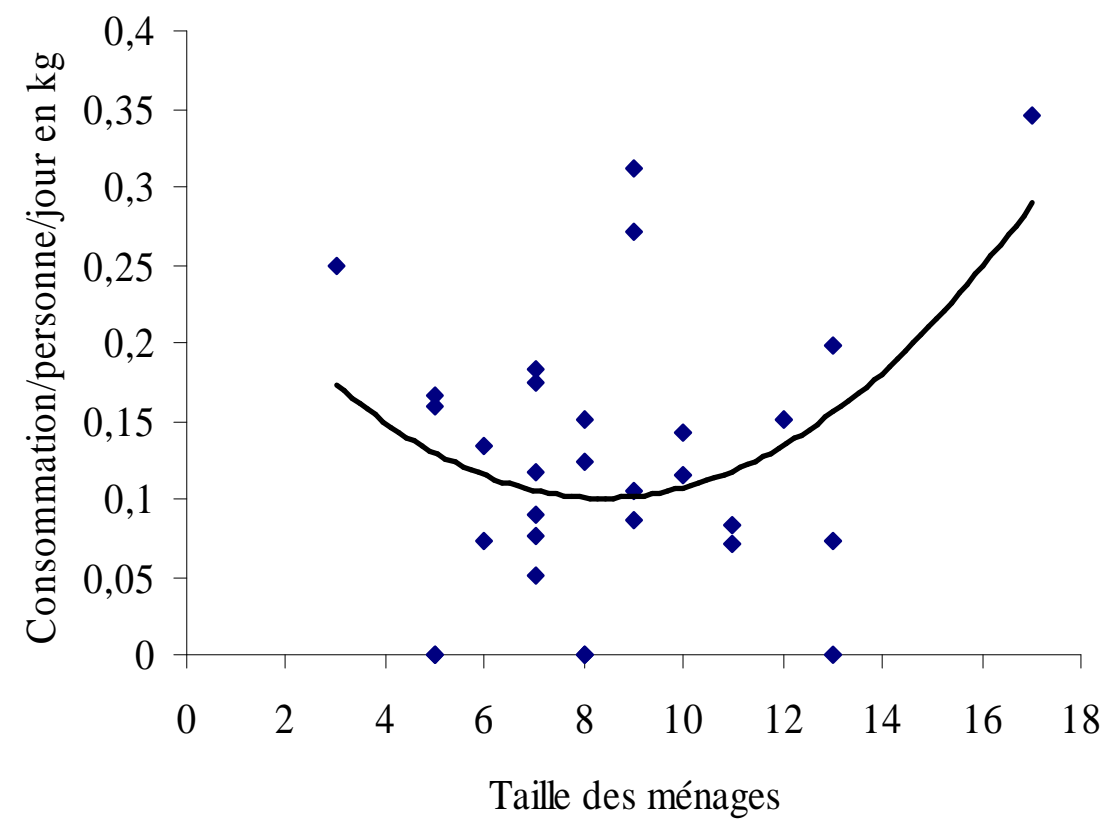

Figure 6: Consommation des résidus de récolte en fonction de la taille du ménage. 


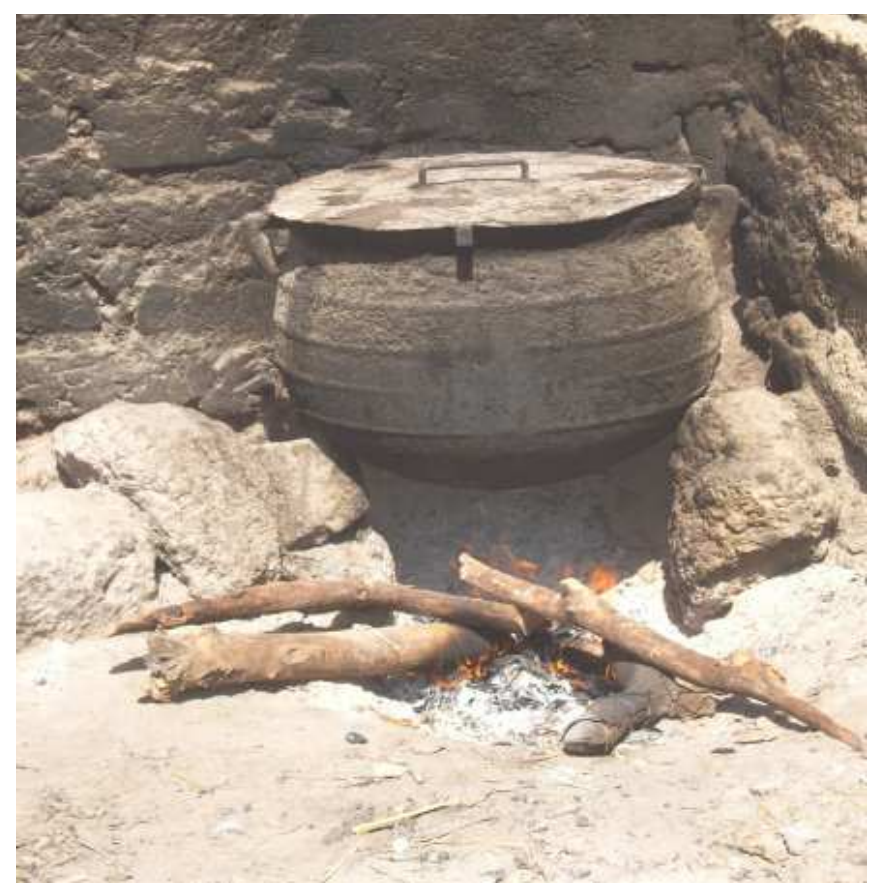

Photo 1: Foyer traditionnel 3 pierres G. Yaméogo 2009.

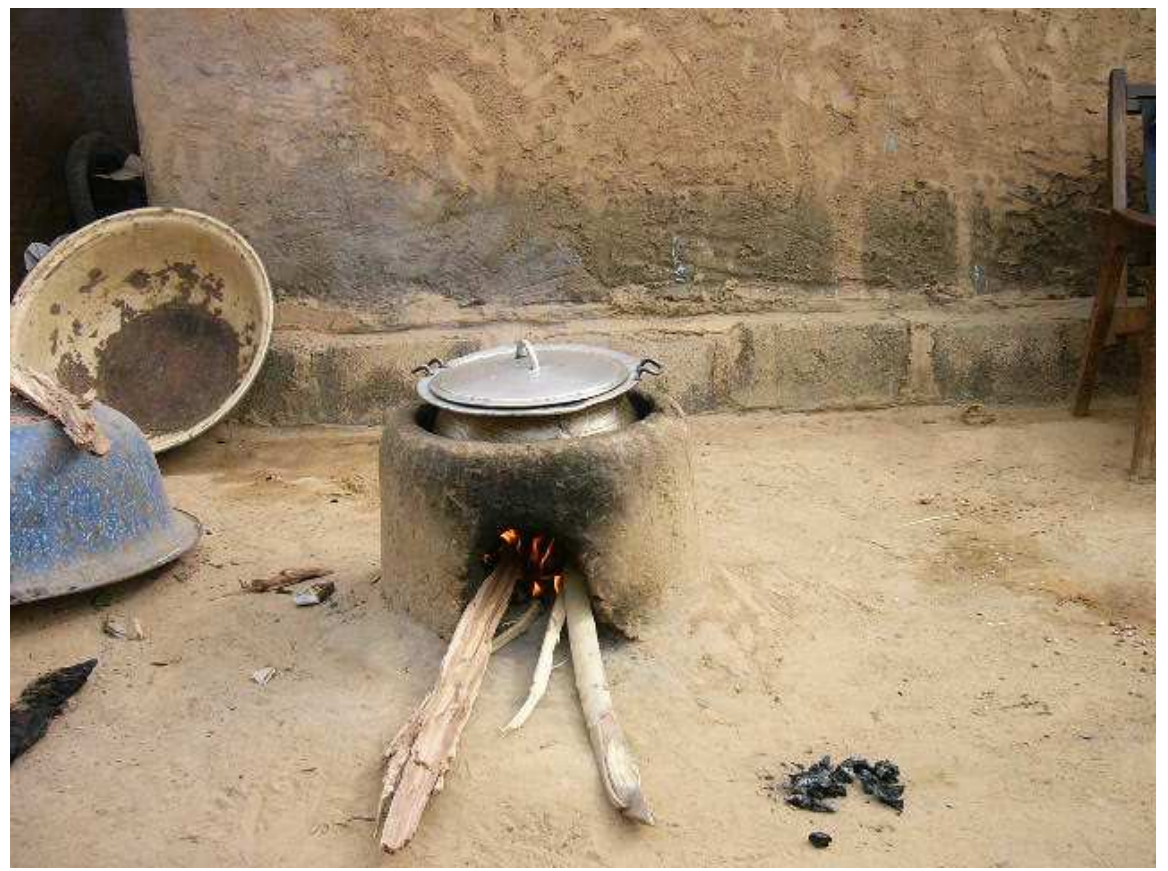

Photo 2: Foyer amélioré : G. Yaméogo 2009. 


\section{DISCUSSION}

La présente étude confirme la place prépondérante des ligneux dans la satisfaction des besoins des populations en bois d'énergie (Yaméogo et al., 2013). Le bois de chauffe contribue pour beaucoup à la satisfaction des besoins énergétiques des ménages à Vipalogo. Ce qui est le cas de manière générale dans la plupart des pays sahéliens (Awaiss et al., 1996 ; Bazile, 1998 ; DGE, 2000). Dans le cas de la présente étude, les besoins journaliers moyens en bois par habitant à Vipalogo sont estimés à 0,64 kg. En milieu rural, au Burkina Faso, selon le SP/CONAGESE (2002), la première estimation faite par la FAO en 1984 a donné un besoin de $1,53 \mathrm{~kg}$ par personne et par jour, mais l'approche utilisée pour cette estimation était plus qualitative que quantitative. A l'échelle du village, les études faites par Damiba (1987) à Séguedin au Burkina Faso font ressortir des consommations journalières par personne de 0,49 kg. Dans le cadre des études commanditées par la Direction Générale de l'Energie (DGE, 2000), la consommation de bois par personne et par jour pour les besoins domestiques en milieu rural a été estimée à $0,94 \mathrm{~kg}$. La méthodologie utilisée pour cette étude est identique à la nôtre. Les travaux effectués par Bazile (1998) au Mali font ressortir un besoin journalier moyen par personne de $0,98 \mathrm{~kg}$ dans le village de Gouani et $0,62 \mathrm{~kg}$ dans le village de N'Goukan. $\mathrm{Au}$ Niger, les valeurs obtenues par Awaiss et al. (1996) varient entre 0,97 et $1 \mathrm{~kg}$ par personne et par jour suivant les villages dans lesquels les études ont été menées. La consommation $\mathrm{du}$ bois de feu par personne et par jour à Vipalogo est inférieure à celle estimée à l'échelle nationale. Cela peut s'expliquer par le fait que dans ce terroir, la pression anthropique est telle qu'on assiste à un amenuisement de la ressource ligneuse, amenant les utilisateurs à prendre des dispositions économiques. Ces dispositions pouvant aller de la conduite du feu à la préparation d'un seul repas chaud par jour.

Quant aux résidus de récolte, leur utilisation pour la satisfaction des besoins des ménages a également été notée par Gautier et al. (2001) au Nord Cameroun. Au niveau national, l'étude de la DGE en 2000 fait ressortir un besoin de $0,01 \mathrm{~kg}$ par personne et par jour, ce qui est en adéquation avec les résultats auxquels nous sommes parvenus dans cette étude. Ce combustible joue la plupart du temps le rôle d'énergie secondaire et vient donc en appoint au bois et cela, sur une période de l'année qui n'excède pas quatre mois. Que ce soit pour le bois ou pour les résidus de récolte, la consommation de combustible dans chaque ménage retenu fait ressortir des variations d'une famille à l'autre et à l'intérieur de la même famille d'un jour à l'autre.

La valeur élevée du coefficient de variation pour les résidus de récolte s'explique par le fait que durant la troisième période de l'étude, ce combustible n'est pas utilisé. Ces valeurs traduisent la complexité de la maîtrise de tous les paramètres au cours des études de terrain et en fonction du type de combustible. Plusieurs raisons peuvent expliquer ces variations :

- le type de repas préparé (habitudes culinaires) et, pour un même type de repas, les ingrédients ajoutés ;

- le type de foyer utilisé: le foyer traditionnel est beaucoup soumis aux aléas climatiques ce qui est encore plus sensible en ce qui concerne les résidus de récolte ;

- la personne qui fait la cuisine (femme de ménage ou fille) ;

- la quantité de repas préparée qui peut varier non seulement en fonction de la taille du ménage mais aussi de certains éléments tels que les activités des membres de la famille, et la saison (bonne ou mauvaise) et ;

- le niveau de revenus du ménage.

En prenant en compte la période de l'année, on constate qu'aussi bien pour le bois 
que pour les résidus de récolte, la période froide est celle pendant laquelle la consommation est la plus élevée en termes de valeur absolue même si statistiquement elle ne diffère pas de celle de la période chaude. C'est également à ce moment que l'on note la plus grande variabilité de la consommation par personne et par jour.

En considérant la variable taille du ménage, la dispersion des nuages de points laisse voir qu'il n'y pas une forte liaison entre la taille et la consommation de combustible. Cela est confirmé par les valeurs des coefficients de corrélation (Damiba, 1987; Bazile, 1998). Les valeurs obtenues à Vipalogo sont toutefois plus faibles que celles des études effectuées par Damiba (1987) à Séguédin et par Bazile (1998) à Gouani. Il se dégage donc que la taille du ménage n'est pas le seul facteur pouvant influencer la consommation de combustibles et que les raisons évoquées plus haut occupent également une place importante.

$\mathrm{Si}$ les résidus de récolte proviennent des champs de culture, le bois provient pour la plupart des formations naturelles qui sont soit des savanes, soit des jachères n'ayant pas été remises en culture depuis plus de cinq ans. Le prélèvement dans les formations naturelles pour les besoins énergétiques occasionne une forte dégradation du milieu, surtout autour des zones d'habitation, ce qui contribue à l'éloignement des zones d'approvisionnement comme on peut le constater sur les cartes d'occupation des terres du terroir. L'éloignement des zones de coupe entraîne des conséquences malheureuses pour les femmes et les enfants à qui incombe la collecte du bois de chauffe.

A partir des différentes réponses, il ressort que les méthodes de collecte et de transport de bois de feu à usage domestique n'ont pas changé. Il est coupé à la main et transporté sur la tête par les femmes et les enfants. DGE (2000), fait également le constat, de même que Benjaminsen (1996) dans le Gourma malien. L'éloignement des lieux de collecte associé à la raréfaction commence à donner une valeur marchande au bois dans le terroir de Vipalogo où on constate que certains ménages achètent le bois pour la satisfaction de leurs besoins énergétiques (Yaméogo et al., 2013).

Pour ce qui est de l'utilisation des espèces ligneuses, on peut dire qu'en dehors de neuf espèces citées par les populations, tout le reste peut être utilisé comme bois de feu. Toutefois, il existe à ce niveau des préférences. La raison de ces préférences serait liée à la disponibilité et également à la qualité de la combustion de leur bois. Quant aux espèces non utilisées, les raisons évoquées sont l'apparition de maladies liées à leur utilisation ainsi que des interdits.

En ce qui concerne les périodes pendant lesquelles les combustibles sont les plus utilisés, l'étude a permis de mettre en évidence que c'est pendant la période froide que les ménages consomment le plus d'énergie quel que soit le combustible considéré. Toutefois, à travers les résultats des analyses statistiques, il n'y a pas de différence significative entre la consommation de bois en période froide et en période chaude alors que pendant cette période, en plus des besoins pour la cuisson, il y a ceux liés au chauffage (eau, maison). Cet état de fait peut être mis en relation avec l'utilisation des résidus de récolte comme énergie d'appoint. La période pluvieuse est celle où la consommation est la moins élevée et l'une des principales raisons est la période de soudure que connaissent les populations rurales à cette époque de l'année.

\section{Conclusion}

A Vipalogo, le bois représente la principale source d'énergie des ménages et est beaucoup utilisé sur toutes les périodes de l'année. L'usage des tiges de mil en appoint comme énergie par les ménages, intervient surtout au cours de la période froide de l'année. Comme dans la plupart des terroirs au 
Burkina Faso, les ressources ligneuses sont fortement sollicitées dans le terroir de Vipalogo pour la satisfaction des besoins énergétiques. Ces ressources énergétiques n'étant pas illimitées, on assiste de plus en plus à une forte dégradation du fait de la pression anthropique et également de l'aridification climatique.

Les populations développent alors des modes d'adaptation à cette situation. Ces nouveaux comportements des populations se traduisent par la collecte et la conservation des résidus de récolte pour la cuisson, parfois par une seule préparation des mets par jour sans réchauffage avant consommation et quelquefois par la réduction du temps de cuisson ou l'adhésion à l'utilisation des foyers améliorés. Cependant, il faut noter que les difficultés d'accéder au combustible énergétique introduit de plus en plus la « mauvaise alimentation», ce qui, à long terme, pourrait causer des problèmes de santé publique.

Il apparaît alors la nécessité de planter des essences à croissance rapide à des fins de combustibles et au développement de sources énergétiques alternatives (gaz, biogaz, solaire, etc.) afin de mieux conserver nos ressources forestières.

\section{REFERENCES}

Awaiss A, Illia MH, Soumana I, Amoukou IA. 1996. Les ressources ligneuses et leur exploitation comme bois de feu et de service à Mayahi. In Gestion des Terroirs et des Ressources Naturelles au Sahel. Actes du séminaire, 2-3 avril 1996, Montpellier, France, CNEARC, pp. 28-23.

Bazile D. 1998. La gestion des espèces ligneuses dans l'approvisionnement en énergie des populations. Cas de la zone soudanienne du Mali. Thèse de doctorat, Université de Toulouse-Le Mirail, Toulouse, $340 \mathrm{p}$.
Benjaminsen TA. 1996. Bois-énergie, déboisement et sécheresse au Sahel: le cas du Gourma malien. Sécheresse, 3(7): 179-185.

Damiba TE. 1987. Consommation du bois de feu dans le village de Séguédin: Disponibilité de la ressource et possibilités d'amélioration de son utilisation. Mémoire de fin d'études, IDR, Option Eaux et forêts, Université de Ouagadougou, Ouagadougou, 124 p.

DGE. 2000. Base de données énergétiques du Burkina Faso. Rapport sur les énergies traditionnelles, $45 \mathrm{p}$.

Edjolo A, Smektala G, Chery JP, Peltier R. 2003. Modélisation systémique de la dynamique de la ressource arborée en zone soudano sahélienne. Le cas du terroir villageois de Maffa Kilda, Nord Cameroun. Actes de colloque, 25-27 février 2003, Montpellier, France, 9 p.

FAO. 1994. Directives pour la Description des Sols $\left(3^{\text {ème }}\right.$ édition révisée). FAO: Rome; 71.

Fontes J, Guinko S. 1995. Carte de la végétation et de l'occupation du sol au Burkina Faso (Notice explicative). Projet Campus. IDR, Université de Ouagadougou, $67 \mathrm{p}$.

Ganaba S, Guinko S. 1995. Morphologie et rôle des structures racinaires dans la mortalité de Pterocarpus lucens Lpr. dans la région sahélienne de la mare d'Oursi (Burkina Faso). Études sur la Flore et la végétation du Burkina Faso et des pays avoisinants, II, pp. 15-24.

Gautier D, Tebaya O, Nounga E, Mana J. 2001. Femmes du Nord Cameroun et Ressources arborées : usages, perception de l'évolution et perspectives d'actions dans cinq villages de référence du PRASAC, Maroua, Cameroun, 110p.

Gueye I, Laban P. 1992. Des bois de villages à la gestion du terroir villageois en Afrique de l'ouest. Programme réseaux des zones arides, dossier $n^{\circ} 35,23 p$. 
INSD. 1998. Recensement général de la population 1985. Structure par sexe des villages du Burkina Faso, INSD. 330 p.

Le Roy E, Karsenty A, Bertrand A. 1996. La sécurité foncière en Afrique. Pour une gestion viable des ressources renouvelables. Karthala, 392 p.

Loireau M. 1998. Espaces-RessourcesUsages: Spécialisation des interactions dynamiques entre les systèmes sociaux et les systèmes écologiques au Sahel nigérien. Thèse de Doctorat, Université Paul Valery Montpellier III, Montpellier, $393 \mathrm{p}$.

SP/CONAGESSE, 2002. Rapport sur l'état de l'Environnement du Burkina Faso. $1^{\text {ère }}$
Edition, Ouagadougou, Burkina Faso. $174 \mathrm{p}$.

Yaméogo, G. 2009. Les ressources ligneuses et leur gestion dans le terroir de Vipalogo, province du Kadiogo, Burkina Faso. Mémoire de thèse Unique de Doctorat, Université de CocodyAbidjan, Abidjan, 224 p.

Yaméogo G, Yélémou B, Kaboré O, Traoré D. 2013. Bois d'énergie du 'dolo' et bois de service: deux modes de consommation de bois à Vipalogo au Burkina Faso. Bulletin de la Recherche Agronomique du Bénin, 73: 1-12. 